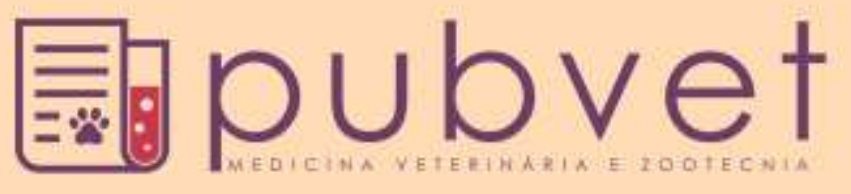

https://doi.org/10.22256/pubvet.v12n1a4.1-5

\title{
Infecção natural por Cytauxzoon felis em onça parda (Puma concolor) de vida livre proveniente da região sudoeste de Mato Grosso do Sul, Brasil
}

\author{
Tamires Ramborger Antunes ${ }^{6} 1 *$, Alexandre Welzel da Silveira ${ }^{\natural}$, Gustavo Gomes de \\ Oliveira ${ }^{6}$, Andressa Salles Rezende ${ }^{6}$, Lucas Bezerra da Silva Azuaga ${ }^{6}$, Michelli \\ Lopes de Souza $^{6}$, Desireé Reis de Oliveira ${ }^{6}$, Claudia Regina Macedo Coutinho Netto ${ }^{5}$, \\ Kelly Cristina da Silva Godoy ${ }^{16}$, Carlos Alberto do Nascimento Ramos ${ }^{6}{ }^{7}$, Alda Izabel \\ de Souza ${ }^{6}$ \\ ${ }^{I}$ Médica Veterinária. Doutoranda do Programa de Pós-Graduação em Ciências Veterinárias, Faculdade de Medicina Veterinária e Zootecnia, \\ Universidade Federal de Mato Grosso do Sul, Campo Grande, MS, Brasil. \\ ${ }^{2}$ Médico Veterinário. Mestrando do Programa de Pós-Graduação em Ciência Animal, Faculdade de Medicina Veterinária e Zootecnia, \\ Universidade Federal de Mato Grosso do Sul, Campo Grande, MS, Brasil. \\ ${ }^{3}$ Médico/a Veterinário/a. Mestrando/a do Programa de Pós-Graduação em Ciências Veterinárias, Faculdade de Medicina Veterinária e \\ Zootecnia), Universidade Federal de Mato Grosso do Sul, Campo Grande, MS, Brasil. \\ ${ }^{4}$ Médica Veterinária. Residente do Laboratório de Patologia Clínica Veterinária, Faculdade de Medicina Veterinária e Zootecnia, \\ Universidade Federal de Mato Grosso do Sul, Campo Grande, MS, Brasil. \\ ${ }^{5}$ Médico/a Veterinário/a, Centro de Reabilitação de Animais Selvagens, Campo Grande, MS, Brasil. \\ ${ }^{6}$ Médica Veterinária. Residente do setor de Diagnóstico por Imagem, Faculdade de Medicina Veterinária e Zootecnia, Universidade Federal \\ de Mato Grosso do Sul, Campo Grande, MS, Brasil. \\ ${ }^{7}$ Professorla da Faculdade de Medicina Veterinária e Zootecnia, Universidade Federal de Mato Grosso do Sul, Campo Grande, MS, Brasil. \\ E-mail: carlosanramos@yahoo.com.br; alda.izabel@ufms.br. \\ Autor para correspondência, E-mail: tamires_ramborger_antunes@hotmail.com
}

RESUMO. Vários fatores como desmatamento e expansão da agricultura e pecuária tem promovido o contato próximo entre animais selvagens e domésticos, levando ao compartilhamento de patógenos, dentre eles o Cytauxzoon felis. O objetivo com este trabalho é relatar um caso de cytauxzoonose em Puma concolor de vida livre proveniente da região sudoeste de Mato Grosso do Sul, Brasil. O diagnóstico foi obtido por meio de microscopia de sangue periférico e reação em cadeia da polimerase, que neste relato, caracterizou-se como achado acidental, uma vez que manifestações clínicas associadas à doença não foram observadas. A detecção de Cytauxzoon felis alerta para a necessidade de estabelecer a importância epidemiológica dos animais selvagens e o risco que representam para as populações domésticas e selvagens de cativeiro em relação aos patógenos transmitidos por carrapatos.

Palavras chave: cytauxzoonose, diagnóstico, piroplasmídeos, felino selvagem

\section{Natural infection by Cytauxzoon felis in brown ounce (Puma concolor) of free life from the southwest region of Mato Grosso do Sul, Brazil}

ABSTRACT. Several factors such as deforestation and expansion of agriculture and
livestock has promoted the close contact between wild and domestic animals, leading to
the sharing of pathogens, including the Cytauxzoon felis. The aim of this study is to report
a case of cytauxzoonose in a free-living Puma concolor from the southwest region of Mato
Grosso do Sul, Brazil. The diagnosis was obtained through microscopy of peripheral blood
smear and polymerase chain reaction, that in this report, was characterized as accidental
finding, since clinical manifestations associated to disease were not observed. The 
detection of Cytauxzoon felis highlights the need to establish the epidemiological importance of wild animals and the risk that they represent to domestic and captive wild populations in relation to tick-borne pathogens transmitted.

Keywords: cytauxzoonosis, diagnosis, piroplasmids, wild feline

\title{
Infección natural por Cytauxzoon felis en onza parda (Puma concolor) de vida libre proveniente de la región suroeste de Mato Grosso do Sul, Brasil
}

\begin{abstract}
RESUMEN. Varios factores como la deforestación y la expansión de la agricultura y la ganadería han promovido el contacto cercano entre animales salvajes y domésticos, llevando al intercambio de patógenos, entre ellos el Cytauxzoon felis. El objetivo con este trabajo es relatar un caso de cytauxzoosis en Puma concolor de vida libre proveniente de la región suroeste de Mato Grosso do Sul, Brasil. El diagnóstico se obtuvo mediante la microscopía de sangre periférica y la reacción en cadena de la polimerasa, que en este relato se caracterizó como hallazgo accidental, ya que las manifestaciones clínicas asociadas a la enfermedad no se observaron. La detección de Cytauxzoon felis alerta sobre la necesidad de establecer la importancia epidemiológica de los animales salvajes y el riesgo que representan para las poblaciones domésticas y salvajes de cautiverio en relación con los patógenos transmitidos por garrapatas.
\end{abstract}

Palabras clave: cytauxzoosis, diagnóstico, piroplasmideo, felino salvaje

\section{Introdução}

Puma concolor (Linnaeus, 1771), conhecida como onça parda, suçuarana ou leão da montanha, é uma espécie de mamífero terrestre da família Felidae e ordem Carnívora (Wilson \& Reeder, 2005), encontrada em diferentes países do continente americano e, no Brasil. Exemplares são visualizados em todos os biomas (Amazônia, cerrado, pantanal, caatinga, mata atlântica e pampa), com exceção de áreas densamente povoadas (Cubas et al., 2014).

O desmatamento, a implementação de áreas de mineração, terras agrícolas e de pastagem para o gado, assim como atropelamentos e a caça ilegal, principalmente em regiões próximas a fazendas tem ameaçado a conservação da onça parda que é considerada vulnerável à extinção no Brasil (Chiarello et al., 2008, Azevedo et al., 2013, Cubas et al., 2014). Além disso, a expansão da agricultura e pecuária tem promovido o contato próximo entre animais selvagens e domésticos, devido à redução do habitat de espécies nativas (Williams et al., 2002), facilitando a transmissão de patógenos, principalmente por meio de vetores artrópodes (Pesquera et al., 2015).

A infecção natural de felídeos ferais, de diferentes espécies, por piroplasmídeos, transmitidos por pulgas e carrapatos, tem sido relatada tanto em animais de cativeiro quanto de vida livre (Garner et al., 1996, Jakob \& Wesemeier, 1996, Soares et al., 2004, Peixoto et al., 2007). Cytauxzoon felis, protozoário da família Teileriidae, é um dos agentes que tem sido descrito nesses indivíduos. Na onça parda, os métodos moleculares são os mais frequentemente utilizados para a identificação deste parasito, sendo escassa, especialmente no Brasil, a descrição de sua visualização em esfregaço de sangue periférico. Portanto, o objetivo com este trabalho é relatar um caso de cytauxzoonose em Puma concolor de vida livre infectado naturalmente cujo diagnóstico inicial foi obtido por meio de detecção parasitológica em esfregaço sanguíneo.

\section{Relato de caso}

Uma onça parda (Puma concolor) jovem, macho, com histórico de atropelamento foi resgatada entre as cidades de Tacuru $\left(23^{\circ} 37^{\prime} 58^{\prime \prime}\right.$ $\left.\mathrm{S}, 55^{\circ} 0^{\prime} 57^{\prime \prime} \mathrm{W}\right)$ e Sete Quedas $\left(23^{\circ} 58^{\prime} 12^{\prime \prime} \mathrm{S}, 55^{\circ}\right.$ $2^{\prime} 9^{\prime \prime}$ W), Mato Grosso do Sul (MS), e encaminhada para o Centro de Reabilitação de Animais Selvagens (CRAS) de Campo GrandeMS. Exames clínicos, radiológicos e hematológicos demonstraram respectivamente: edema de membros anteriores; fratura cominutiva de úmero em membro torácico e entre púbis e acetábulo esquerdo, além de fissura na tábua isquiática direita; leucocitose por neutrofilia e 
piroplasmídeos intraeritrocitários compatíveis com Cytauxzoon felis (Figura 1A).

Procedimento cirúrgico para redução das fraturas, colocando-se pinos intramedulares e Reação em Cadeia da Polimerase (PCR) (Figura 1B) para determinação da espécie do piroplasmídeo detectado, utilizando-se o protocolo descrito Brown et al. (2010) foram realizados.

Devido à gravidade das fraturas e o desalinhamento dos pinos intramedulares, provocado pela movimentação do animal, o mesmo foi eutanasiado, de acordo com a legislação vigente no Brasil.

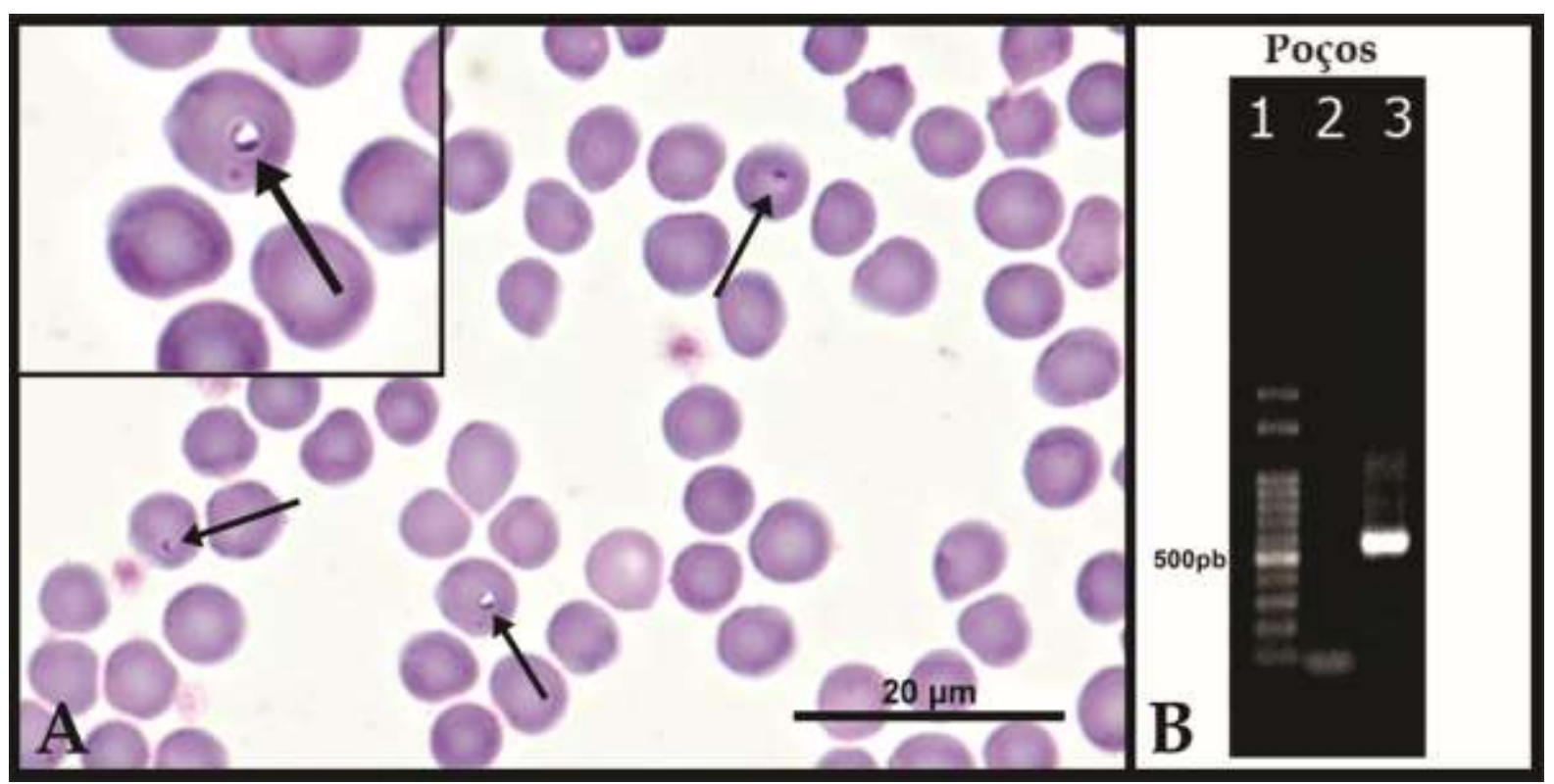

Figura 1. A. Esfregaço de sangue periférico. Inclusões intraeritrocitárias compatíveis com Cytauxzoon felis (setas). Em destaque o piroplasmídeo para melhor visualização. Objetiva de 100x, barra: $20 \mu \mathrm{m}$, coloração do tipo hematológica rápida. B. Resultado de amplificação por PCR de amostra de sangue periférico de Puma concolor demonstrando reação positiva para Cytauxzoon felis. Poço 1 - Marcador de pares de base "Low Molecular Weight DNA Ladder" (New England Biolabs); Poço 2 - Controle negativo; Poço 3 - Amostra de DNA extraída do sangue periférico de Puma concolor.

\section{Discussão}

A cytauxzoonose em gatos domésticos, em algumas regiões do mundo, caracteriza-se por apresentar evolução aguda e geralmente fatal. Os principais sinais clínicos visualizados incluem pirexia, anorexia, desidratação e depressão, e morte entre 19-21 dias pós-infecção (Wagner et al., 1980). No entanto, a sobrevivência de indivíduos tratados e não tratados já foi descrita (Motzel \& Wagner, 1990, Haber et al., 2007). No Brasil, infecções assintomáticas têm sido mais frequentemente identificadas, inclusive em gatos domésticos provenientes de Campo Grande, Mato Grosso do Sul (André et al., 2015).

Em felinos selvagens, a enfermidade é clinicamente variável, com quadros de infecção persistente e assintomática e/ou evolução fatal, em diferentes espécies. No presente relato, nenhuma manifestação clínica compatível com a cytauxzoonose foi observada, corroborando com os achados de Soares et al. (2004) que detectaram o agente em uma onça parda, e André et al. (2009) que identificaram material genético do protozoário em seis jaguatiricas (Leopardus pardalis), duas onças pardas e uma onça pintada (Panthera onca). Em contrapartida, doença fatal em leões (Panthera leo), tigre de bengala e tigre branco (Panthera tigres) foi descrita (Garner et al., 1996, Jakob \& Wesemeier, 1996, Peixoto et al., 2007)

A presença desse patógeno, na ausência de sinais clínicos, pode estar associada à eficácia do sistema imune, no entanto, a hipótese de uma estirpe menos patogênica envolvida na infecção ou, a possibilidade de que o Puma concolor atue, assim como o Lynx rufus, como hospedeiro reservatório natural da doença deve ser investigada (Meinkoth et al., 2000, Soares et al., 2004).

A leucocitose por neutrofilia, identificada na análise hematológica, provavelmente resulta do processo inflamatório em consequência das fraturas apresentadas pelo animal, uma vez que felina com cytauxzoonose clínica têm 
demonstrado citopenia como achado laboratorial (Harvey et al., 2007). Na reação inflamatória, as células mononucleares presentes no tecido liberam citocinas que estimulam a medula óssea a aumentar a taxa de produção e liberação de neutrófilos maduros para o sangue. Devido ao consumo ser inferior à liberação medular, o aumento do número de neutrófilos circulantes é observado (Stockham \& Scott, 2011).

A detecção parasitológica de Cytauxzoon felis em esfregaço sanguíneo tem sido relatada em felinos da América do Norte. Em um levantamento envolvendo panteras da Flórida (Puma concolor coryi) e pumas do Texas (Puma concolor stanleyana), ambos de vida livre, a eritroparasitemia foi identificada em aproximadamente $36 \%$ dos animais (Rotstein et al., 1999).

No Brasil, descrição da identificação do agente em extensões sanguíneas é escassa e foi relatada apenas por Soares et al. (2004) e assim como no presente relato, caracterizou-se como achado acidental devido à ausência de manifestações clínicas.

Infecção por Cytauxzoon felis foi confirmada em 32\% (38/118) dos gatos domésticos que frequentavam um zoológico do Rio de Janeiro-RJ (Mendes-de-Almeida et al., 2007). Estes números sinalizam que a presença do parasito em animais de vida livre capturados possibilita a transmissão de patógenos para espécies selvagens em reabilitação no CRAS ou para felinos domésticos que coabitam a região.

\section{Conclusão}

A presença do agente em esfregaço de sangue periférico indica a possibilidade da utilização do hemograma como forma auxiliar para investigação de infecções em animais selvagens, mesmo na ausência de manifestações clínicas, sendo necessária a realização de estudos que determinem a eficácia deste método.

A detecção do Cytauxzoon felis, no presente relato, alerta para a necessidade de estabelecer o papel epidemiológico de felinos silvestres e o risco oferecido às populações domésticas e selvagens em cativeiro.

\section{Agradecimentos}

A Fundação de Apoio ao Desenvolvimento do Ensino, Ciência e Tecnologia do Estado de Mato
Grosso do Sul (FUNDECT) pelas bolsas de estudos concedidas.

\section{Referências Bibliográficas}

André, M. R., Adania, C. H., Machado, R. Z., Allegretti, S. M., Felippe, P. A. N., Silva, K. F., Nakaghi, A. C. H. \& Dagnone, A. S. 2009. Molecular detection of Cytauxzoon spp. in asymptomatic Brazilian wild captive felids. Journal of Wildlife Diseases, 45, 234-237.

André, M. R., Herrera, H. M., Jesus Fernandes, S., Sousa, K. C. M., Gonçalves, L. R., Domingos, I. H., Macedo, G. C. \& Machado, R. Z. 2015. Tick-borne agents in domesticated and stray cats from the city of Campo Grande, state of Mato Grosso do Sul, midwestern Brazil. Ticks and Tick-Borne Diseases, 6, 779-786.

Azevedo, F. C., Lemos, F. G., Almeida, L. B., de Campos, C. B., Mello Beisiegel, B., Paula, R. C., Junior, P. G. C., Barros, K. M. P. M. \& Oliveira, T. G. 2013. Avaliação do risco de extinção da onça-parda Puma concolor (Linnaeus, 1771) no Brasil. Biodiversidade Brasileira, 107-121.

Brown, H. M., Lockhart, J. M., Latimer, K. S. \& Peterson, D. S. 2010. Identification and genetic characterization of Cytauxzoon felis in asymptomatic domestic cats and bobcats. Veterinary Parasitology, 172, 311-316.

Chiarello, A. G., Aguiar, L. M. S., Cerqueira, R., Melo, F. R., Rodrigues, F. H. G. \& Silva, V. M. F. 2008. Mamíferos ameaçados de extinção no Brasil. Livro vermelho da fauna brasileira ameaçada de extinção, 2, 680-880.

Cubas, Z. S., Silva, J. C. R. \& Dias, J. L. C. 2014. Tratado de animais selvagens-medicina veterinária. Editora Roca.

Garner, M. M., Lung, N. P., Citino, S., Greiner, E. C., Harvey, J. W. \& Homer, B. L. 1996. Fatal cytauxzoonosis in a captive-reared white tiger (Panthera tigris). Veterinary Pathology, 33, 82-86.

Haber, M. D., Tucker, M. D., Marr, H. S., Levy, J. K., Burgess, J., Lappin, M. R. \& Birkenheuer, A. J. 2007. The detection of Cytauxzoon felis in apparently healthy free-roaming cats in the USA. Veterinary Parasitology, 146, 316-320.

Harvey, J. W., Dunbar, M. R., Norton, T. M. \& Yabsley, M. J. 2007. Laboratory findings in acute Cytauxzoon felis infection in cougars (Puma concolor couguar) in Florida. Journal of Zoo and Wildlife Medicine, 38, 285-291. 
Jakob, W. \& Wesemeier, H. H. 1996. A fatal infection in a Bengal tiger resembling cytauxzoonosis in domestic cats. Journal of Comparative Pathology, 114, 439-444.

Meinkoth, J., Kocan, A. A., Whitworth, L., Murphy, G., Fox, J. C. \& Woods, J. P. 2000. Cats surviving natural infection with Cytauxzoon felis: 18 cases (1997-1998). Journal of Veterinary Internal Medicine, 14, 521-525.

Mendes-de-Almeida, F., Labarthe, N., Guerrero, J., Faria, M. C. F., Branco, A. S., Pereira, C. D., Barreira, J. D. \& Pereira, M. J. S. 2007. Follow-up of the health conditions of an urban colony of free-roaming cats (Felis catus Linnaeus, 1758) in the city of Rio de Janeiro, Brazil. Veterinary Parasitology, 147, 9-15.

Motzel, S. L. \& Wagner, J. E. 1990. Treatment of experimentally induced cytauxzoonosis in cats with parvaquone and buparvaquone. Veterinary Parasitology, 35, 131-138.

Peixoto, P. V., Soares, C. O., Scofield, A., Santiago, C. D., Franca, T. N. \& Barros, S. S. 2007. Fatal cytauxzoonosis in captive-reared lions in Brazil. Veterinary Parasitology, 145, 383-387.

Pesquera, C., Portillo, A., Palomar, A. M. \& Oteo, J. A. 2015. Investigation of tick-borne bacteria (Rickettsia spp., Anaplasma spp., Ehrlichia spp. and Borrelia spp.) in ticks collected from Andean tapirs, cattle and vegetation from a protected area in Ecuador. Parasites \& Vectors, 8, 46.

Rotstein, D. S., Taylor, S. K., Harvey, J. W. \& Bean, J. 1999. Hematologic effects of cytauxzoonosis in Florida panthers and Texas cougars in Florida. Journal of Wildlife Diseases, 35, 613-617.

Soares, J. R., Souza, A. I. \& Netto, N. T. 2004. Cytauxzoon felis-like in mountain lion (Puma concolor): a case report. Journal of Animal and Veterinary Advances, 3, 820-823.

Stockham, S. L. \& Scott, M. A. 2011. Fundamentos de patologia clínica veterinária, Rio de Janeiro.

Wagner, J. E., Ferris, D. H., Kier, A. B., Wightman, S. R., Maring, E., Morehouse, L. G. \& Hansen, R. D. 1980. Experimentally induced cytauxzoonosis-like disease in domestic cats. Veterinary Parasitology, 6, 305-311.

Williams, E. S., Yuill, T., Artois, M., Fischer, J. \& Haigh, S. A. 2002. Emerging infectious diseases in wildlife. Revue Scientifique et Technique-Office International des Epizooties, 21, 139-158.

Wilson, D. E. \& Reeder, D. M. 2005. Mammal species of the world: a taxonomic and geographic reference. Johns Hopkins University Press, Rio de Janeiro, Brasil.

Article History:

Received 16 August 2017

Accepted 13 September 2017

Available on line 21 October 2017

License information: This is an open-access article distributed under the terms of the Creative Commons Attribution License 4.0, which permits unrestricted use, distribution, and reproduction in any medium, provided the original work is properly cited. 